\title{
Targeted hypoglossal neurostimulation for obstructive sleep apnoea: a 1-year pilot
} study

\author{
Gimbada B. Mwenge*,\#, Philippe Rombaux*,9, Myriam Dury\#, \\ Benoît Lengelé $^{+}$and Daniel Rodenstein ${ }^{\star, \#}$
}

ABSTRACT: Continuous positive airway pressure (CPAP) is an effective but cumbersome treatment for obstructive sleep apnoea (OSA). Noncompliant patients need alternative therapies.

We studied a tongue neurostimulation approach: targeted hypoglossal neurostimulation (THN) therapy with the aura $6000^{\mathrm{TM}}$ System. A multi-contact electrode positioned around the main trunk of the twelfth nerve connected to an implanted pulse generator stimulates segments of the nerve, activating dilator muscles. The primary objective was to improve the polysomnographically determined apnoea/hypopnoea index (AHI) at 3 months, and maintain the improvement after 12 months of treatment. 13 out of 14 operated patients were successfully implanted.

At 12 months, the AHI decreased from $45 \pm 18$ to $21 \pm 17$, a $53 \%$ reduction $(p<0.001)$. The $4 \%$ oxygen desaturation index fell from $29 \pm 20$ to $15 \pm 16$ and the arousal index from $37 \pm 13$ to $25 \pm 14$, both $p<0.001$. The Epworth sleepiness scale decreased from $11 \pm 7$ to $8 \pm 4(p=0.09)$. THN was neither painful nor awakened patients, who all complied with therapy. There were two transient tongue paresis.

The present study represents the longest study of any hypoglossal neurostimulation reported to date. We conclude that THN is safe and effective to treat OSA in patients not compliant with CPAP.

KEYWORDS: Obstructive sleep apnoea, sleep apnoea, sleep apnoea diagnosis, sleep apnoea treatment, sleep-disordered breathing, sleep medicine

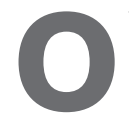

bstructive sleep apnoea (OSA) is characterised by repetitive episodes of respiratory arrest despite continuing breathing efforts. This condition only occurs during sleep and is due to complete or severe, though incomplete, pharyngeal collapse secondary to the sleep-related decrease in pharyngeal neuromuscular activity $[1,2]$. Each apnoea/hypopnoea results in two main consequences: 1) decrease in oxygen saturation; and 2) sleep interruption by short arousals (termed microarousals) allowing the resumption of breathing [3]. When these episodes repeat $>15-20$ times per hour of sleep, clinical consequences may arise, including fatigue, daytime sleepiness, irritability, cognitive impairment, nocturia and arterial hypertension $[4,5]$. When the apnoea/hypopnoea index (AHI) is $>30$ ( $>30$ events $\cdot \mathrm{h}^{-1}$ of sleep), OSA can lead to myocardial infarctions, cerebrovascular accidents, vehicle crashes and premature deaths [6-11].
OSA is readily treated with continuous positive airway pressure (CPAP) applied during sleep, all night and every night, through the nares [12]. The positive pressure pushes apart the walls of the pharynx, allowing the subject to breathe, and hence to sleep [12]. Many patients accept this cumbersome treatment, with immediate and long-lasting relief of their symptoms [13]. However, a number of patients are unable to adapt to CPAP and remain untreated [14]. Alternative treatments (avoidance of supine decubitus in positional OSA, mandibular advancement devices or surgical modifications of the pharyngeal airway) may be useful in mild OSA, but are of little help in moderate or severe OSA [13]. The only proven effective alternative is a tracheostomy, which is seldom considered nowadays. Therefore, the search for new therapeutic options remains necessary.

\section{AFFILIATIONS}

*Dept of Pneumology, Cliniques universitaires Saint-Luc, Université catholique de Louvain,

\#Center for Sleep Medicine,

Cliniques universitaires Saint-Luc, Université catholique de Louvain,

"Dept of Otholaryngology, Cliniques universitaires Saint-Luc, Université catholique de Louvain, and +Dept of Plastic Surgery, Cliniques universitaires Saint-Luc, Université catholique de Louvain, Brussels, Belgium.

\section{CORRESPONDENCE}

D. Rodenstein

Dept of Pneumology and Center for Sleep Medicine

Cliniques universitaires Saint-Luc, Av. Hippocrate 10

1200 Brussels

Belgium

E-mail daniel.rodenstein@

uclouvain.be

Received:

March 092012

Accepted after revision:

May 012012

First published online:

May 172012 
Electrical stimulation of pharyngeal muscles is a promising alternative to keep the pharyngeal airway open throughout sleep, counteracting the sleep-related decrease in muscle tone. The hypothesis that stimulation of the genioglossus could effectively dilate the pharynx was first tested in the late 1990s and, more recently, in two different industry-sponsored studies [15-18]. However, the genioglossus is part of the tongue, a complex structure with intrinsic and extrinsic muscles behaving like a hydrostat [19]. The assumption that the genioglossus can be stimulated in isolation, without eliciting co-activation of other extrinsic and intrinsic tongue muscles, is probably an oversimplification. Indeed, on the one hand, part of the genioglossus horizontal segment inserts into the hyoid bone [20], and its activation will therefore modify the force balance that determines the hyoid position with consequent variable reactions from the styloglossus and hyoglossus muscles. On the other hand, a change in volume of the tongue due to contraction of the genioglossus will lead to a change in shape depending on the variable activity of the intrinsic tongue muscles, which cannot be predicted from the simple stimulation of the genioglossus alone [21]. It is conceivable that stimulating not one but several tongue muscles with a net pharyngeal enlarging effect could achieve resolution of apnoeas and hypopnoeas. As all tongue muscles are innervated by the hypoglossal nerve, it might be possible to target several muscles with a net favourable effect by selective stimulation in the proximal portion of the hypoglossal nerve, before it branches. This report presents the results of a 12-month safety and efficacy study of this concept.

\section{MATERIALS AND METHODS}

\section{Stimulation device}

The aura $6000^{\mathrm{TM}}$ system (ImThera Medical Inc., San Diego, CA, USA) consists of an implanted pulse generator (IPG), a small implant containing the battery and stimulation system (hardware and software), and a multi-electrode lead with an 8-mm soft silicone cuff housing six independent electrodes, connected to the IPG via a subcutaneously tunnelled lead wire. The IPG is implanted in the upper chest, in a subcutaneous pocket, whereas the electrode cuff is furled around the hypoglossal nerve near the middle tendon of the digastric muscle, so that the six stimulating electrodes are radially in contact with the cylindrical body of the proximal hypoglossal nerve.

The IPG battery is rechargeable. Recharging is performed transcutaneously with an external remote control charger (RCC) and charging coil that is placed over the IPG with the help of two magnets. The charging time is $\sim 1 \mathrm{~h}$. The same RCC is used to start, pause and end each night session of stimulation. The IPG has a log memory to record actual charging and use.

\section{Implantation surgical procedure}

The surgical procedure was performed under general anaesthesia, and started with the hypoglossal nerve dissection. The cuff electrode was rolled under and around the main trunk of the nerve (fig. 1a) and the electrode was looped and anchored nearby. Thereafter, the subcutaneous pectoral pocket was created and the IPG implanted. Using a liposuction cannula a subcutaneous tunnel was created between the two incisions. The lead was passed downwards and connected to the IPG. The electrical integrity was confirmed and the incisions closed.
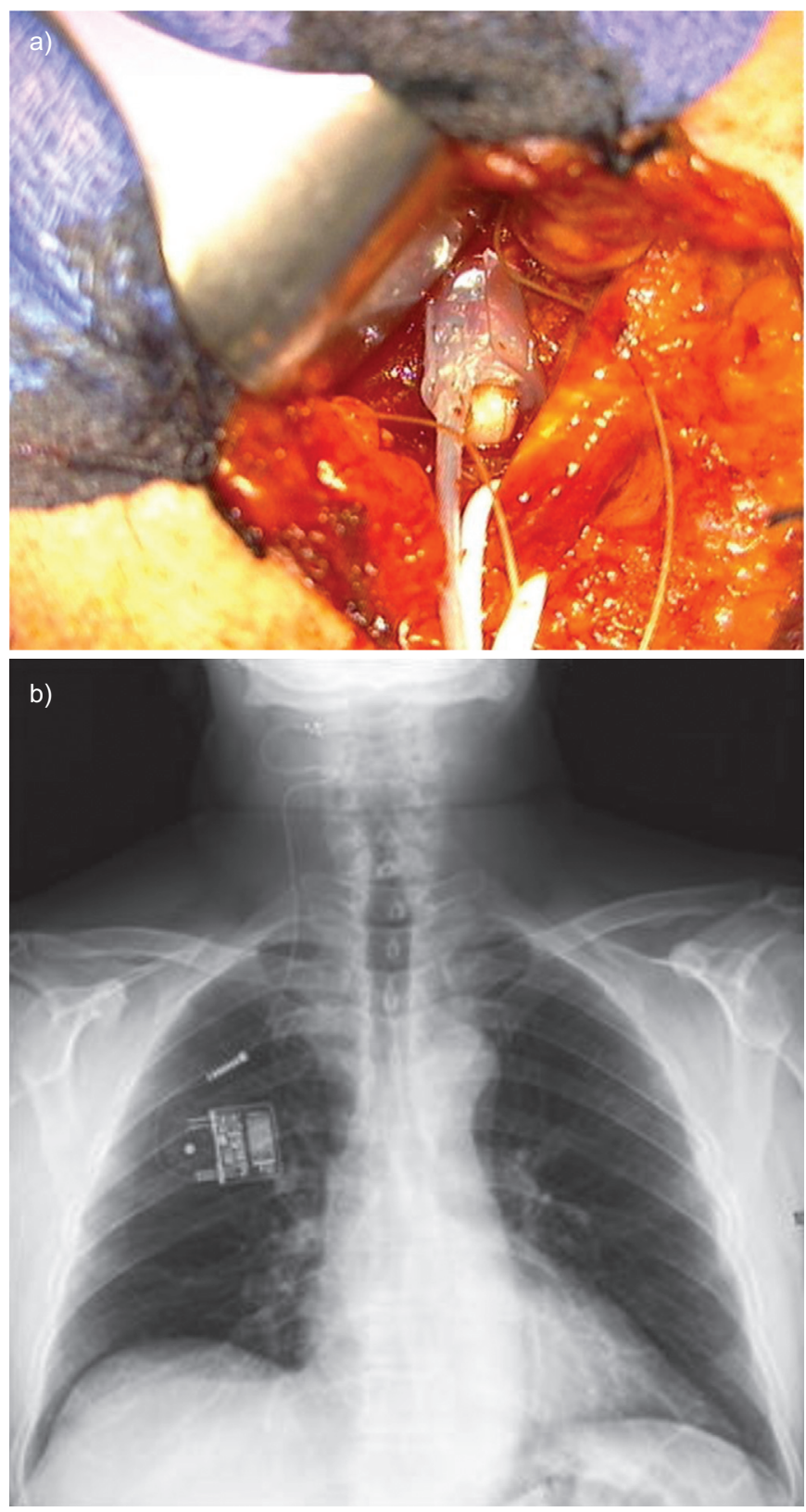

FIGURE 1. a) Surgical detail showing the hypoglossal nerve with the electrode cuff furled around its main trunk. The white band at the bottom of the figure is lowering the middle tendon of the digastric muscle. b) Thoracic radiograph showing the implantable pulse generator, connector and electrode cuffed with six contacts furled around the hypoglossal nerve.

Figure 1 shows a radiological view of the implanted system in one patient.

\section{Stimulation protocol}

Initially, stimulation was titrated in seated awake patients 34 weeks after surgery. Each contact was stimulated until the patients felt a painless sensation (sensory threshold). Thereafter, each contact was again stimulated until bulk movement was observed via pharyngeal fibreoptic endoscopy (motor threshold). In most patients, there was a ventral movement of the base 

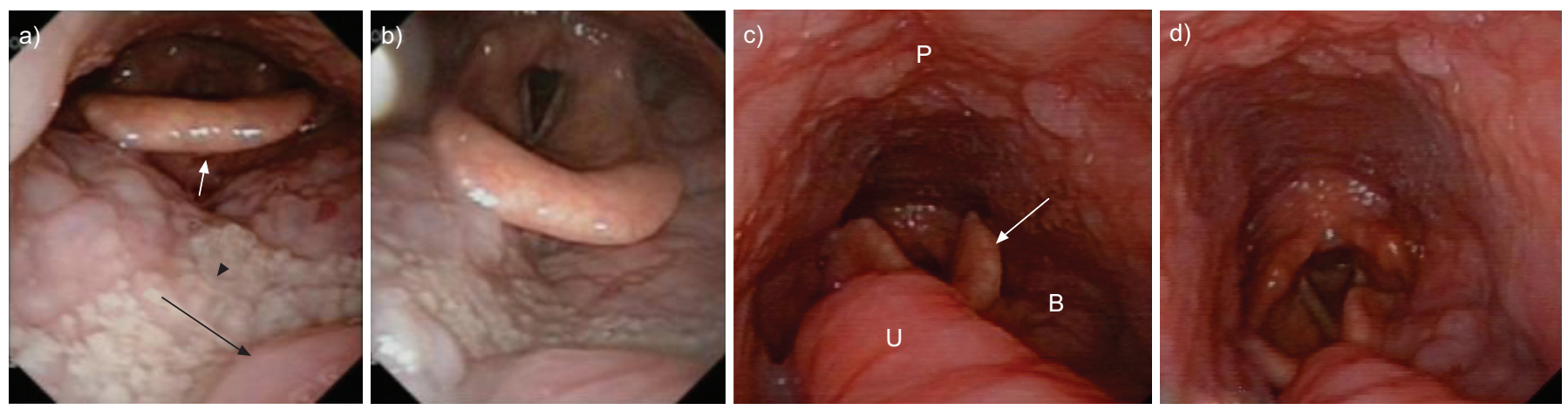

FIGURE 2. Endoscopic view of the pharynx in two patients, seen from the oropharynx downwards. a, b) Patient implanted on the left side. The posterior pharyngeal wall is on top; the base of the tongue (arrowhead) with apposed uvula (black arrow) on the bottom. The epiglottis is seen in-between (white arrow). a) Natural breathing without stimulation and $b$ ) the same area during stimulation. The left hemitongue projects anteriorly, pulling with it the epiglottis. The change in voice of this patient during stimulation while repeating "un, deux, trois, quatre" [one, two, three, four] can be heard in the online supplementary material; a video of the pharynx during stimulation can also be seen. c, d) Patient implanted on the right side. The posterior pharyngeal wall $(P)$ is on top, and the base of the tongue (B) on the bottom. The uvula (U) is particularly thick and long c) Natural breathing without stimulation and d) the same area during stimulation: the right hemitongue projects forward, pulling the epiglottis with it and enlarging the pharynx.

of the tongue pulling with it the epiglottis (fig. 2 and the online supplementary video-recording) and, in a minority of cases, we saw a stiffening of the hemipharynx with little displacement. The contacts with greatest effect were selected for stimulation therapy. Patients then underwent the first stimulation polysomnography (PSG).

Titration was repeated before the final 12-month recordings, this time with patients semi-recumbent, and with the use of $2 \mathrm{mg}$ midazolam to facilitate relaxation (and frequently sleep). The tongue bulk movements were assessed at the pharynx through fibreoptic pharyngoscopy and within the mouth by placing the tip of the fibreoptic endoscope at the entrance of the mouth with patients wearing a mouthpiece (fig. 3).

A detailed description of the device, stimulation technique and surgical procedure are given in the supplementary material.

\section{Study design}

This was an open-label, single-site, single-arm safety and efficacy study in patients with untreated moderate-to-severe OSA due to intolerance to CPAP. The device and protocol were approved by the Belgian Federal Medicines and Medical Devices Agency (Agence Fédérale de Médicaments et Produits de Santé), and the Université catholique de Louvain Ethics Committee, Brussels, Belgium. The study was registered at www.clinicaltrials.gov (NCT01532180).

\section{Outcomes}

The primary safety measure was the number and type of adverse events (AEs). We recorded AEs at each visit, as well as at the time of their occurrence. Patients had full access to the medical and surgical team throughout the study. AEs were classified as serious and simple adverse events. We added a
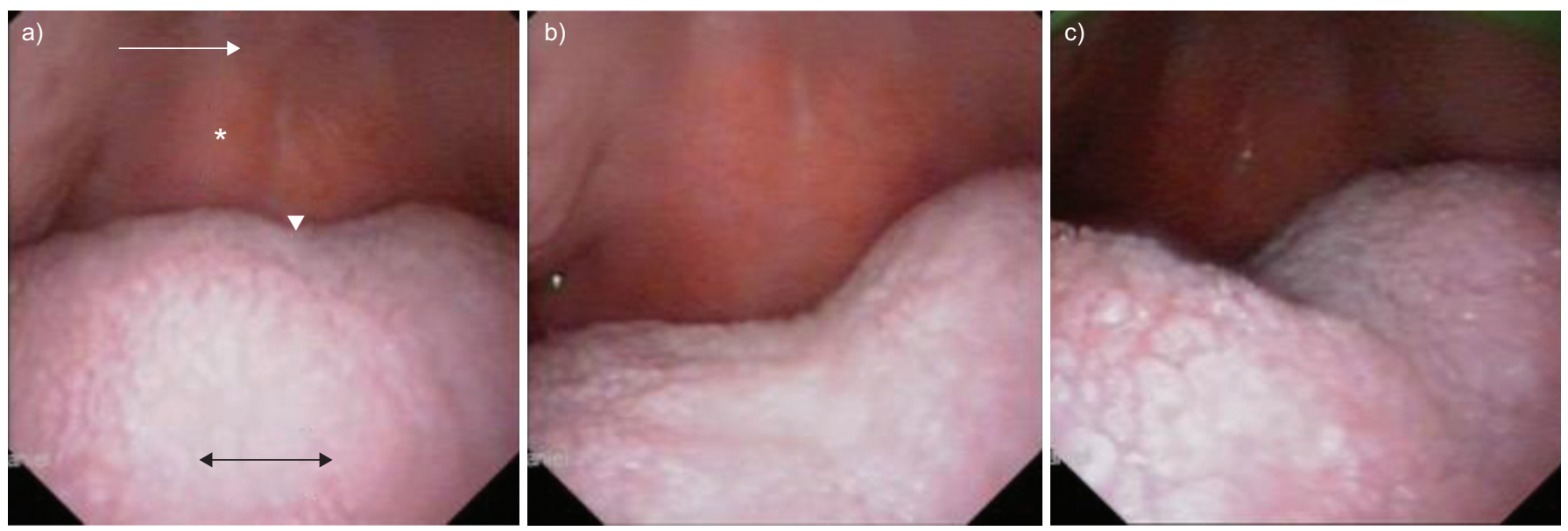

FIGURE 3. Example of different motions of the oral tongue according to stimulation through different contacts. a) Oral tongue at rest. The white arrow signals the hard palate, the asterisk signals the soft palate, the arrowhead shows the lingual medial sulcus. The black arrow shows the oral tongue. b) Useful stimulation. Right hemitongue stimulation results in flattening and forward motion, enlarging the tongue-soft palate space (this should facilitate nasal and oral breathing). c) Useless stimulation. Right hemitongue stimulation in the same patient with a different contact results in deepening of the medial sulcus without forward movement, and without any improvement in pharyngeal space. 
category of technical AEs, related to the device and affecting patient management but not causing untoward health consequences in themselves.

The primary efficacy measure was the mean change in $\mathrm{AHI}$ at polysomnographies performed 3 and 12 months relative to presurgical baseline. Secondary outcomes included mean changes from baseline in the oxygen desaturation index (ODI), the Epworth sleepiness scale, the fatigue severity scale and parameters of sleep quantity and quality.

\section{Patient selection}

We selected patients suffering from full PSG-confirmed OSA who were eligible for CPAP treatment reimbursement according to the Belgian Social Security rules, which require an AHI of at least 20 events per hour of sleep. Patients had used CPAP in the past and had either stopped treatment or refused to use CPAP altogether. Some patients had already failed various surgical treatments (table 1). All patients gave written consent before the start of the study.

Inclusion criteria consisted of baseline AHI $\geqslant 20$ events $\cdot h^{-1}$, refusal of CPAP treatment, body mass index (BMI) 25$40 \mathrm{~kg} \cdot \mathrm{m}^{-2}$, age $25-70 \mathrm{yrs}$, modified Mallampati score from I to III and palatine tonsils assessed as grade 0,1 or 2 [18]. There was no preferential selection of subjects for apnoea or hypopnoea indices. Exclusion criteria included pregnancy, central sleep apnoea (CSA), diagnosis of restless leg syndrome or insomnia, presence of a syndromic craniofacial abnormality, clinically enlarged tonsils (grade 3 or 4), modified Mallampati score of IV, presence of obstructive nasal polyps, current alcohol or drug abuse, psychiatric disorders, and subjects unable to give a valid informed consent or to comply with follow-up requirements.

\section{Polysomnography}

Full-night PSG included the recording of three channels of electroencephalogram, two electrooculograms, one chin electromyogram channel and one lead electrocardiogram. Snoring was obtained from a microphone glued to the patient's neck. Airflow was assessed with a nasal cannula for nasal flow, and with an oronasal thermocouple for oral flow and as a backup nasal signal. Two inductive uncalibrated elastic bands monitored thoracic and abdominal respiratory movements. Body position was estimated from a sensor placed above the sternum, and oxygen saturation and pulse rate were obtained from a finger sensor of a pulse oximeter. Periodic legs movements were computed from two piezoelectric sensors placed on the right and left ankles (Medatec BrainNet, Brussels, Belgium). The polysomnographies were analysed by hand according to Rechtschaffen and Kales, with stages 3 and 4 nonrapid eye movement sleep merged. Microarousals were scored according to the American Academy of Sleep Medicine, and reported as the number of microarousals per hour of sleep or microarousal index. Apnoeas and hypopnoeas were identified according to the American Academy of Sleep Medicine rules, with hypopnoeas defined as a $\geqslant 30 \%$ decrease in the flow signals lasting $\geqslant 10 \mathrm{~s}$ and inducing either $\mathrm{a} \geqslant 4 \%$ oxygen saturation fall or a microarousal. The number of apnoeas and hypopnoeas per hour of sleep is given as the AHI. The percentage of apnoeas and hypopnoeas in the supine position is also reported. The ODI was defined as the number of falls in oxygen saturation $\geqslant 4 \%$ per hour of sleep.

\section{Study course}

Patients were seen 1 week after surgery for evaluation of the surgical sites and recording of AEs. The first titration procedure was performed $\sim 1$ month after surgery, followed

TABLE 1 Patients' anthropometric data, and medical and sleep history

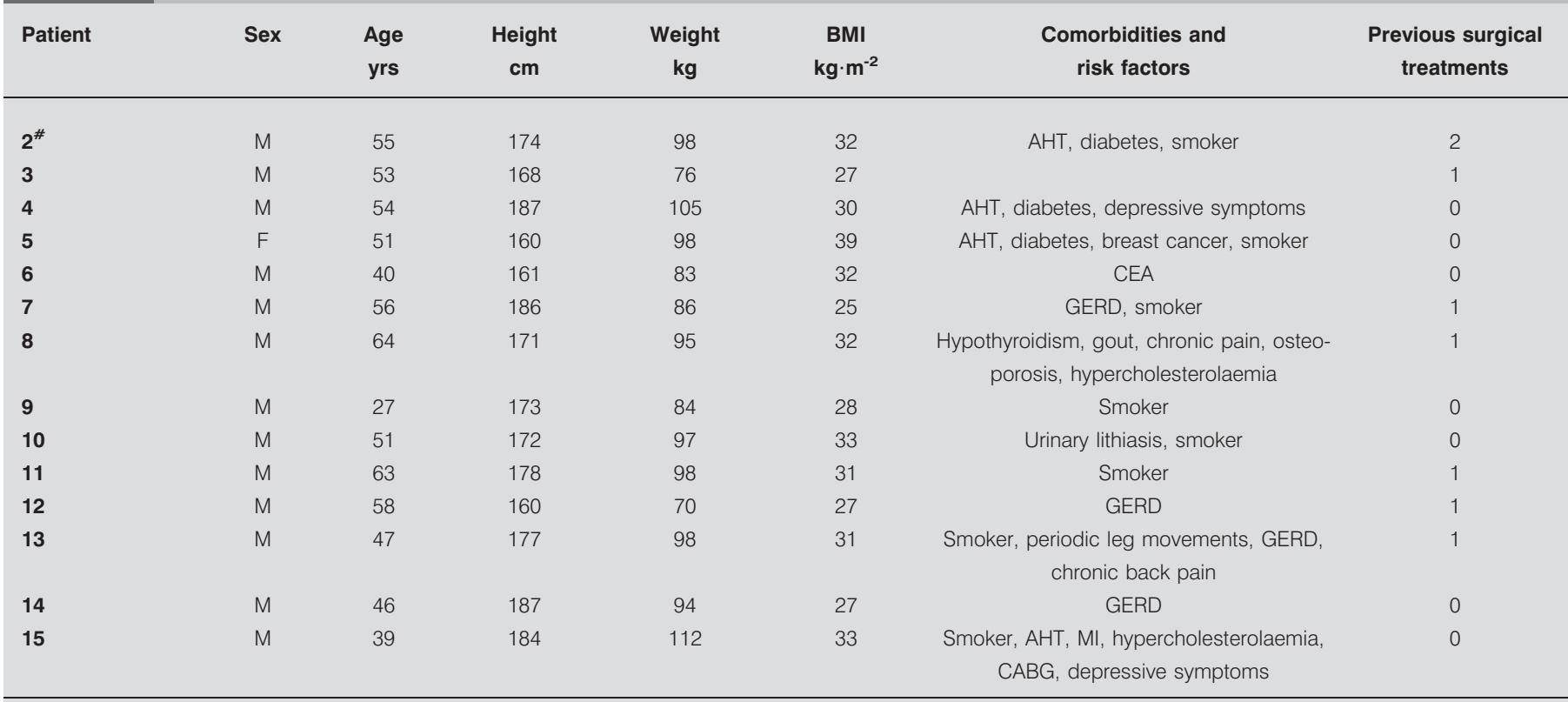

BMI: body mass index; M: male; F: female; AHT: arterial hypertension; CEA: congenital oesophageal atresia (corrected); GERD: gastro-oesophageal reflux; MI: myocardial

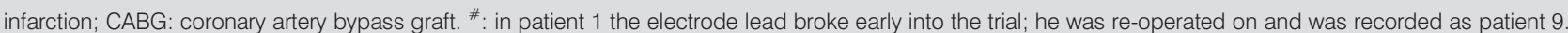


by a first PSG with stimulation parameter adjustments. During the night, stimulation parameters were further adjusted aiming to decrease residual apnoeas and hypopnoeas. This involved changing the stimulation current, the contact stimulation time, the stimulation frequency or the cathodic phase duration on the previously selected contacts. Changes sometimes succeeded in improving the situation. At other times the changes induced arousal with painful sensations. Patients were discharged home with this first set of parameters and instructed to start daily treatment. Patients used the dedicated RCC to initiate a sleep session, followed by a 45-min delay before stimulation started for a pre-determined 7-h duration. They were then seen at regular visits until the third month, where a new PSG was performed. Again, stimulation parameters were adjusted during sleep to improve residual events, and patients left the following morning with the new settings. Again, patients were seen at regular intervals and whenever they needed between scheduled visits. AEs were always sought and recorded, and when time permitted the compliance log was downloaded. Final data were recorded at 12 months, after a new titration, during a final PSG.

\section{Statistical analysis}

Data are presented as mean \pm SD. Statistical differences between baseline and both follow-up visits were assessed using a linear, repeated measures regression model. To accommodate model assumptions, a logarithmic transformation was necessary for the AHI, the arousal index, the ODI and sleep latency. A compound symmetric covariance matrix was adequate to model the inter-subject variability for all end-points. To determine if a significant number of patients responded to treatment at both 3 and 12 months, Chi-squared tests for equal binomial proportions were performed; $\mathrm{p}$-values $<0.05$ were considered to be of statistical significance. All statistical analyses were conducted in SAS Version 9.2 (SAS Institute, Cary, NC, USA), and were performed by an independent consultant (INTEGRA Group, Brooklyn Park, MN, USA).

\section{RESULTS}

\section{Patients}

14 patients (one female) took part in the study. Their main anthropometric characteristics, comorbidities and history related to OSA are described in table 1.13 patients had moderate-tosevere OSA and one patient with previously diagnosed severe OSA presented with both obstructive and central apnoeas (he had been implanted with a morphine pump for chronic back pain and was consuming hypnotics at the time of the study).

\section{Acute surgical results}

13 subjects were successfully implanted. One subject could not be included in the study because of a defective IPG connector discovered during surgery. All materials were then explanted, and the subject recovered without harm but did not participate further in the study. The IPG was implanted on the right side in all but one subject. This subject had a right-side Port-a-Cath ${ }_{\mathbb{B}}$ (Smiths Medical, St Paul, MN, USA). 10 subjects were discharged the day after surgery, whereas three subjects in whom wound drainage persisted were kept in hospital for an extra day. Pain was well controlled with simple pain medicines. All subjects could drink and eat during the evening after surgery, and all subjects could speak and had control of tongue movements on arrival to the ward. Mean surgical time was $110 \mathrm{~min}$ (range 85-145 min).

\section{Main outcomes}

Figures 2 and 3 show the type of motion we observed during titration, with enlargement of the pharyngeal airway and flattening of the oral hemitongue. Motion of both tongue base and oral tongue should facilitate nasal and oral breathing. Video images and voice sound changes induced by stimulation can be obtained from the online supplementary material. Figure 3 shows that different contacts can stimulate different nerve fibres and lead to different motions of the tongue, some enlarging the pharynx and palate-lingual space, while others having no beneficial effect. Table 2 shows the main outcome data. In all 13

\section{TABLE 2 Change in sleep parameters}

\begin{tabular}{|c|c|c|c|c|c|}
\hline Parameter & Baseline & 3 months & p-value & 12 months & p-value \\
\hline \multicolumn{6}{|l|}{ Sleep-disordered breathing } \\
\hline $\mathrm{AHI}$ events $\cdot h^{-1}$ & $45.2 \pm 17.8$ & $21.7 \pm 19.9$ & $<0.001$ & $21.0 \pm 16.5$ & $<0.001$ \\
\hline Micro-arousals events $\cdot h^{-1}$ & $36.8 \pm 12.5$ & $24.9 \pm 14.4$ & $<0.001$ & $24.9 \pm 13.7$ & 0.001 \\
\hline Oxygen desaturation index events $\cdot \mathrm{h}^{-1}$ & $29.2 \pm 19.6$ & $14.2 \pm 16.7$ & $<0.001$ & $15.3 \pm 16.2$ & 0.001 \\
\hline \multicolumn{6}{|l|}{ Sleep architecture } \\
\hline Stage 1 NREM sleep \% TST & $10.6 \pm 6.1$ & $8.4 \pm 4.5$ & 0.190 & $8.7 \pm 7.1$ & 0.467 \\
\hline Stage 2 NREM sleep \% TST & $67.2 \pm 7$ & $67.8 \pm 8.1$ & 0.766 & $64.5 \pm 12$ & 0.478 \\
\hline Stages 3-4 NREM sleep \% TST & $5.2 \pm 5.6$ & $7.2 \pm 5.3$ & 0.243 & $9.2 \pm 9.1$ & 0.188 \\
\hline Stage REM \% TST & $17.2 \pm 5.6$ & $16.7 \pm 6.2$ & 0.804 & $17.9 \pm 5.6$ & 0.731 \\
\hline
\end{tabular}

Data are presented as mean $\pm \mathrm{SD}$, unless otherwise stated. AHI: aponoea/hypopnoea index; TST: total sleep time; NREM: nonrapid eye movement; REM: rapid eye movement. 
a)

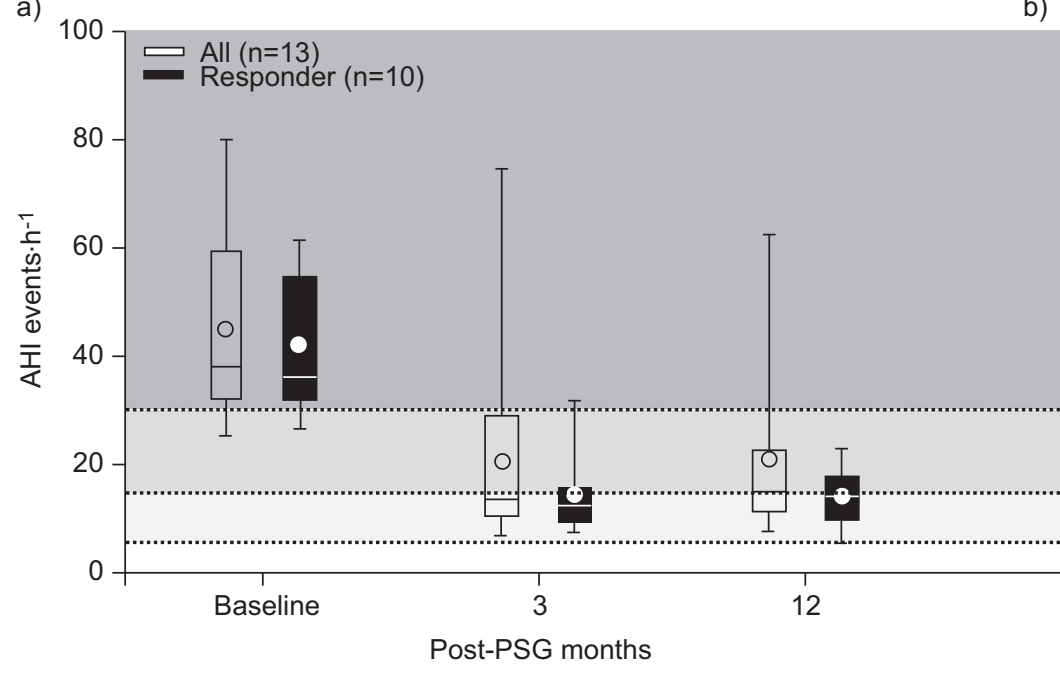

b)

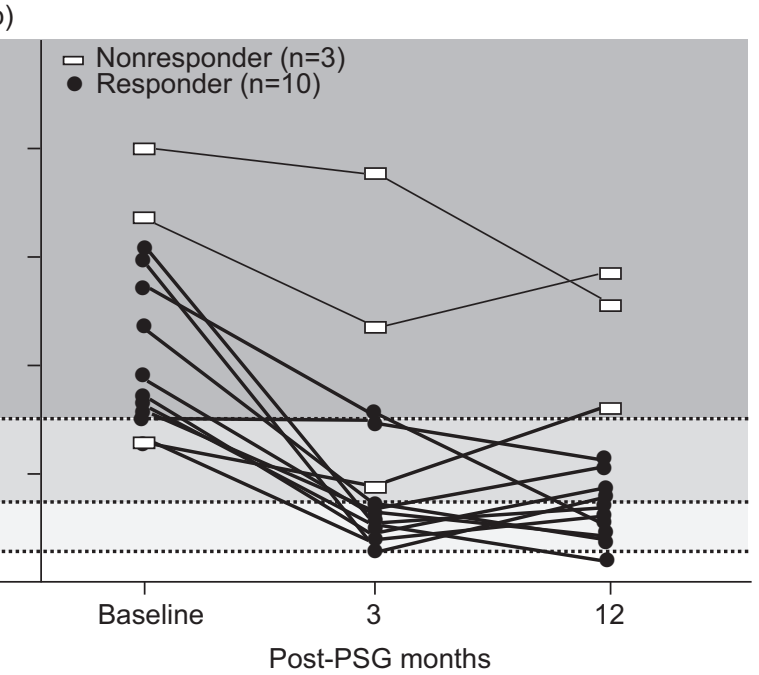

FIGURE 4. Apnoea/hypopnoea index (AHI) scores at baseline, and at 3 and 12 months post-polysomnography (PSG) for all patients. a) Boxplots of all subjects and responder groups showing the median (interquartile range). The open circle indicates the median values. Whiskers represent the maximum value (top) and the minimum value (bottom) of the dataset. b) Line graphs showing individual data of all 13 patients. The graduated shades represent the distribution of AHI in mild, moderate and severe obstructive sleep apnoea (bottom to top, respectively).

subjects, AHI decreased significantly at 12 months from $45.2 \pm 17.8$ (baseline) to $21.0 \pm 16.5$ (table 1 and fig. 4), the ODI decreased from $29.2 \pm 19.6$ to $15.3 \pm 16.2$ and the arousal index from $36.8 \pm 12.5$ to $24.9 \pm 13.7$. Whereas at baseline only $35 \pm 28 \%$ of apnoeas and hypopnoeas were observed in the dorsal decubitus, the percentage increased at $3(47 \pm 35)$ and 12 $(56 \pm 27)$ months, with a significant difference $(p<0.03)$. Total sleep time did not change throughout the study, with mean values exceeding $400 \mathrm{~min}$ (table 2 ). The BMI did not change throughout the study (initial and final BMI $31 \pm 3 \mathrm{~kg} \cdot \mathrm{m}^{-2}$ ).

We failed to obtain satisfactory clinical results in three subjects. One subject had an unusually large and long uvula (fig. 2c and d), one subject had predominant CSA and, in the last subject (the most severe and obese subject, baseline AHI 80 event $\cdot \mathrm{h}^{-1}$ and BMI $39 \mathrm{~kg} \cdot \mathrm{m}^{-2}$ ), no particular cause was found. A subset analysis was completed excluding these subjects: the mean $\mathrm{AHI}$ for the remaining 10 subjects (responders) decreased from $41.5 \pm 13.1$ to $14.3 \pm 8.8$ at 3 months and $13.2 \pm 5.5$ at 12 months, ODI decreased from $23.1 \pm 10.2$ to $7.6 \pm 4.1$ at 3 months and $7.8 \pm 5.3$ at 12 months, and micro-arousals decreased from $34.8 \pm 6.7$ to $21.0 \pm 9.0$ at 3 months and $20.4 \pm 8.1$ at 12 months. Although the differences between baseline and 3- and 12month data are greater, there is no change in the general trend of the results.

\begin{tabular}{lrrrrr} 
TABLE $\mathbf{3}$ & \multicolumn{6}{c}{ Change in symptoms } \\
Parameter & Baseline & $\mathbf{3}$ months & p-value & $\mathbf{1 2}$ months & p-value \\
\hline \multirow{2}{*}{ ESS } & $10.8 \pm 6.2$ & $6.7 \pm 5.4$ & 0.023 & $7.9 \pm 4.2$ & 0.094 \\
FSS & $4.5 \pm 1.6$ & $3.6 \pm 1.8$ & 0.071 & $3.6 \pm 1.5$ & 0.085 \\
\hline
\end{tabular}

Data are presented as mean $\pm \mathrm{SD}$, unless otherwise stated. ESS: Epworth sleepiness scale; FSS: fatigue severity scale.
Daytime sleepiness decreased significantly at 3 months and showed a tendency to decrease at 12 months, whereas subjective fatigue as measured by the fatigue severity scale (FSS) tended to decrease at both 3 and 12 months (table 3). FSS scores for responders decreased from $4.5 \pm 1.3$ to $3.8 \pm 2.0$ at 3 months and to $3.3 \pm 1.5$ at 12 months. Epworth sleepiness scale scores for responders decreased from $9.4 \pm 4.4$ to $5.6 \pm 5.4$ at 3 months and $7.0 \pm 4.3$ at 12 months.

\section{Safety data}

\section{AES and SAEs}

One patient underwent surgery but could not be implanted due to a defective IPG connector. Two patients experienced transient ipsilateral hemitongue paresis, lasting 2 and 3 months, respectively, with full recovery thereafter. In one patient, this was asymptomatic, whereas the other one could not whistle during this time. No other subjective complaint was recorded due to hemitongue paresis. Post-operative swelling lasted for 2 weeks in one patient. Three leads broke in two patients, one early in the study and two at the end of the study. One patient was reoperated and re-implanted, but his replaced lead broke at the end of the study. One IPG failed by the middle of the study and was replaced, and the patient completed the study. One patient had a Twiddler's phenomenon. The IPG was manually repositioned, and the stimulation continued without further trouble. There were no deaths in the study. No patient asked to exit the study or have the system explanted. All patients, including the nonresponders, continue to use the system. There were no infectious or haemorrhagic complications.

\section{Technical AEs}

All patients experienced one or more technical AEs. The most frequent was transient therapy interruptions due to malfunctions of the external devices used in this study (the RCC and the charging coil), which needed either repair or replacement. All patients could continue into the study after these malfunction events were taken care of. Several patients reported 
uncomfortable stimulation at some point during the study (resolved by reducing the stimulation settings), whereas two patients requested upward adjustments because the stimulation was less well perceived. In three patients, a sleep session started during daytime with an unexpected surprising but painless effect. Supplementary table E1 summarises all AEs.

\section{DISCUSSION}

The application of unilateral targeted hypoglossal neurostimulation (THN) in patients with untreated severe OSA for a 12-month period led to clinically and statistically significant improvements in apnoeas and hypopnoeas, and falls in oxygen saturation and sleep fragmentation, i.e. the main physiopathological events in OSA syndrome. Subjective symptoms of daytime sleepiness and fatigue tended to improve, although they were not abnormal at baseline.

We studied patients with mostly severe OSA. Only two of them had an AHI $<30$ events $\cdot h^{-1}$ (AHI 25 and 26 events $\cdot h^{-1}$, respectively); all others had AHI between 31 and 80 events $\cdot h^{-1}$. Patients were not particularly healthy, having many comorbidities commonly seen in OSA (table 1). However, most patients showed large improvements, with final AHI and ODI results below what can be considered as a serious health risk. The surgical literature on OSA usually classifies patients as either responders or nonresponders based on a $50 \%$ decrease in AHI and/or $>20$ events per hour [17]. This is not at all evidence based. An AHI of 30 may be more relevant, with several longterm mortality studies pointing to it as the frontier between surviving and dying in patients with OSA [6, 10, 11, 20], and constituting the frontier between moderate and severe OSA [22]. If we consider the former classification, nine $(69 \%)$ out of 13 patients were responders. If we consider an AHI of $30,77 \%$ of the patients were responders.

Pilot feasibility studies are generally less restrictive in their exclusion criteria than later studies. Indeed, they enable the identification of patients less likely to respond to a treatment. The two previous pilot industry-sponsored studies conducted to date have reported a lower response rate than the present study. For instance, the seminal paper by EASTWOOD et al. [17] selected patients with predominant hypopnoeas but $<20 \%$ apnoeas. The mean apnoea index in those subjects was 4.8 at baseline whereas, in the present subjects, the figure was 13.4, suggesting that airway obstruction was less severe in the group of EASTWOOD et al. [17]. Similarly, in the pilot study sponsored by Inspire Medical Systems (Maple Grove, MN, USA), only six (30\%) out of 20 patients were responders during the first phase of the study, with responders having a baseline AHI $<30$ [23]. Our subjects showed a higher rate of response. We believe this may be due to a different mechanism of action. Our hypothesis is that the two prior studies sought to stimulate a single protrusor muscle, which could result in antagonistic activation of other tongue muscles with a final net obstructing effect, whereas, in the present study, we sought to stimulate an undetermined number of tongue muscles, eventually both protractor and retractor ones, seeking a final net favourable movement both in the pharynx and mouth, a concept consistent with the hydrostat model of the tongue [19]. Of course, this remains speculative at this time and should be further investigated in future studies. As in the other studies, not all of our subjects responded as expected. Particular characteristics may have affected therapy performance and should be avoided through stricter inclusion criteria in future studies. For instance, patient 5 had the highest BMI $\left(39 \mathrm{~kg} \cdot \mathrm{m}^{-2}\right)$. One may wonder whether morbidly obese patients might require bilateral rather than unilateral stimulation. Patient 8 , initially with pure OSA, had needed the implantation of a morphine pump and was included into the study with both obstructive and central apnoeas. This is certainly not an indication for THN. Patient 10 had an unusually long and thick uvula that perhaps should have been resected before the study.

In contrast to CPAP or to pharmacological therapies, THN requires a surgical approach. This implies by necessity that surgery should have a very low rate of surgical complications, as it is a necessary step but does not in itself constitute the treatment of OSA. We saw no serious surgery-related adverse event, and surgical pain was modest and subsided rapidly. Of the two more serious AEs, hemitongue paresis, one was asymptomatic (patient's tongue tip deviated slightly to the left when protruding the tongue). The other patient's sole symptom as long as the paresis continued was the inability to whistle. Both patients recovered without sequellae. The surgical procedure was rather simple, with the hypoglossal nerve being readily accessible below the digastric muscle. Mean surgery time was $<2 \mathrm{~h}$, despite time devoted to photography and video-recordings, which would not occur in clinical practice. Implantation of the IPG at the thoracic site was simple, facilitated by the small size of the implant. It is our impression that surgeons well trained in ear, nose and throat or maxillofacial surgery should be able to readily perform surgical implantations.

The THN method of hypoglossal nerve stimulation uses cyclical stimulation to insure that no single nerve fibre is continuously stimulated. This should minimise the risk of nerve or muscle fatigue, of which we did not observe any clinical manifestation in this long-term study. Other "closed-loop" systems stimulate during inspiration, requiring extra leads to follow and identify inspiratory time [17]. The THN method appears, in this regard, to be simpler, in that it obviates the need for sensors and extra hardware, thereby decreasing the surgical procedure time and associated risks. Titration was performed first in awake patients. Although this seemed to suffice for the initial procedure, midazolam was used later to allow a longer and more detailed view of the changes in tongue position during stimulation. However, we obtained no further improvement in the results after the last titration.

Patients found the treatment comfortable, and the need to charge the IPG for $1 \mathrm{~h}$ daily did not seem to represent an obstacle to compliance. Charging time and frequency should decrease as new and better batteries become available.

This study had limitations. The study group is small, and thus results could be biased by outliers. This does not seem to be the case when one examines the individual data, shown in figure 4 . The prototype devices needed repairs and changes, but once these were performed all patients could continue into the study, and none of these technical AEs (except the two last electrode breaks) resulted in study interruptions. This needs, of course, to be solved before large-scale studies are undertaken.

The subjective results might seem less impressive than the objective data. However, the subjective results were not abnormal at baseline (a frequent finding in sleep apnoea studies). Therefore, 
there was less room for improvement. By contrast, one of the strengths of our study is its very long-term duration, being the longest study of hypoglossal neurostimulation studies reported to date.

In conclusion, we have found THN to be safe and effective in improving patients with untreated severe OSA who are intolerant to $\mathrm{CPAP}$, allowing most patients to move from a severe to a mild form of the disease. Therapeutic improvement was obtained at 3 months post-implant and maintained at 12 months without signs of adverse effects.

\section{SUPPORT STATEMENT}

This study was supported by ImThera Medical Inc., San Diego, CA, USA.

\section{CLINICAL TRIAL}

This study is registered at clinicaltrials.gov with identifier number NCT01532180.

\section{STATEMENT OF INTEREST}

A statement of interest for the study itself can be found at www.erj. ersjournals.com/site/misc/statements.xhtml

\section{REFERENCES}

1 Gastaut H, Tassinari C, Duron B. Etude polygraphique de manifestations episodiques (hypniques et respiratoires) du syndrome de Pickwick. [Polygraphic study of diurnal and nocturnal (hypnic and respiratory) episodal manifestations of Pickwick syndrome.] Rev Neur (Paris) 1965; 112: 568-579.

2 Dempsey JA, Veasey SC, Morgan BJ, et al. Pathophysiology of sleep apnea. Physiol Rev 2010; 90: 47-112.

3 Remmers JE, DeGroot WJ, Sauerland EK. Pathogenesis of upper airway obstruction during sleep. J Appl Physiol 1978; 44: 931-938.

4 Malhotra A, White DP. Obstructive sleep apnoea. Lancet 2002; 360: 237-245.

5 Duran Cantolla J, Aizpuru F, Montserrat JM, et al. Continuous positive airway pressure as a treatment for systemic hypertension in people with obstructive sleep apnoea: randomised controlled trial. BMJ 2010; 34: c5991.

6 Marin JM, Carrizo SJ, Vicente E. Long term cardiovascular outcomes in men with obstructive sleep apnea with or without treatment with continuous positive airway pressure: an observational study. Lancet 2005; 365: 1046-1053.

7 Yaggi HK, Concato J, Kernan WN, et al. Obstructive sleep apnea as a risk factor for stroke and death. N Eng J Med 2005; 353: 2034-2041.
8 Teran-Santos J, Jimenez-Gomez A, Cordero-Guevara J. The association between sleep apnea and the risk of traffic accidents. Cooperative Group Burgos-Santander. N Eng J Med 1999; 340: 847-851.

9 Rodenstein D. Sleep apnea: traffic and occupational accidents; individual risks, socioeconomic and legal implications. Respiration 2009; 78: 241-248.

10 Marshall NS, Wong KK, Liu PY, et al. Sleep apnea as an independent risk factor for all-cause mortality: The Busselton Health Study. Sleep 2008; 31: 1079-1085.

11 Young T, Finn L, Peppard PE. Sleep disordered breathing and mortality: eighteen-year follow-up of the Wisconsin sleep cohort. Sleep 2008; 31: 1071-1078.

12 Sullivan CE, Issa FG, Berthon-Jones M, et al. Reversal of obstructive sleep apnoea by continuous positive airway pressure applied through the nares. Lancet 1981; i: 862-865.

13 Giles TL, Lasserson TJ, Smith B, et al. Continuous positive airway pressure for obstructive sleep apnoea in adults. Cochrane Database Syst Rev 2006; 3: CD001106.

14 Sucena M, Liistro G, Aubert G, et al. Continuous positive airway pressure treatment for sleep apnoea: compliance increases with time in continuing users. Eur Respir J 2006; 27: 761-766.

15 Schwartz AR, Benett ML, Smith PL, et al. Therepeutic electrical stimulation of the hypoglossal nerve in obstructive sleep apnea. Arch Otolaryngol Head Neck Surg 2001; 127: 1216-1223.

16 Kezirian EJ, Boudawyns A, Eisele D, et al. Electrical stimulation of the hypoglossal nerve in the treatment of obstructive sleep apnea. Sleep Med Rev 2010; 14: 299-305.

17 Eastwood PR, Barnes M, Walsh JH, et al. Treating obstructive sleep apnea with hypoglossal nerve stimulation. Sleep. 2011; 34: 1479-1486.

18 The STAR trial: a research study for obstructive sleep apnea. www. thestartrial.com/index.php/about-the-study Date last updated: July 12, 2012. Date last accessed: October 3, 2012.

19 Kier WM, Smith KK. Tongues, tentacles and trunks: the biomechanics of movement in muscular hydrostats. Zool J Linn Soc 1985; 83: 307-324.

$20 \mathrm{Mu}$ L, Sanders I. Human tongue neuroanatomy: nerve supply and motor endplates. Clin Anat 2010; 23: 777-791.

21 Hiiemae KM, Palmer JB. Tongue movements in feeding and speech. Crit Rev Oral Biol Med 2003; 14: 413-429.

22 Punjabi NM, Caffo BS, Goodwin JL, et al. Sleep-disordered breathing and mortality: a prospective cohort study. PLoS Med 2009; 6: 1-9.

23 Van de Heyning PH, Badr MS, Baskin JZ, et al. Implanted upper airway stimulation device for obstructive sleep apnea. Laryngoscope 2012; 122: 1626-1633. 